\title{
Les relations entre le rythme d'excrétion et la teneur en eau des miellats chez les pucerons Cinara pectinatae (Nördl) et Cinara pilicornis (Hartig) (Homoptera, Lachnidae) et le rythme de récolte de ces miellats par l'abeille domestique Apis mellifica $L$
}

\author{
A Bloc *, H Montagner \\ Université de Besançon, faculté des sciences et des techniques, laboratoire de psychophysiologie, \\ Route de Gray, 25030 Besançon Cedex, France
}

(Reçu le 12 avril 1989; accepté le 15 janvier 1990)

\begin{abstract}
Résumé - L'étude de l'activité de récolte des miellats du puceron Cinara pectinatae (Nördl) par l'abeille domestique, montre qu'il existe des relations entre le rythme de cette activité, le rythme d'excrétion des miellats par le puceron et les variations du pourcentage des miellats en matière sèche tout au long de la journée. Elle révèle précisément comment le rythme d'excrétion des miellats et leur teneur en matière sèche tout au long du nycthémère, constituent des facteurs qui limitent la récolte des miellats par l'abeille domestique.
\end{abstract}

Cinara / puceron / miellat / rythme d'excrétlon / rythme de récolte / Ap/s mellifica

\section{INTRODUCTION}

Les travaux concernant la récolte du nectar des fleurs d'Angiospermes par l'abeille domestique, montrent qu'elle est tributaire du rythme de production du nectar par les nectaires, de la quantité et de la qualité du nectar, et de l'intensité de la miellée (Lundie, 1925; Kleber, 1935; Korner, 1939). Parmi les autres facteurs qui interviennent dans cette récolte, il faut citer la température, l'intensité lumineuse, le vent, les précipitations et la distance entre la ruche et les aires de butinage (Ribbands, 1953).

En revanche, il n'y a pas d'étude précise sur les facteurs qui règlent la récolte des miellats de pucerons à partir desquels les abeilles élaborent, dans la ruche, les miels dits de sapin. C'est pourquoi, nous avons entrepris de cerner les facteurs temporels (rythmes de récolte) et les facteurs microclimatiques (température et hygrométrie) qui interviennent dans la régulation de ce comportement.

Tout d'abord, d'observations préliminaires sur le déroulement d'une miellée de sapin (Abies alba Mill) et sur le comportement de récolte du miellat par l'abeille, il ressort que l'activité de récolte des abeilles fluctue au cours de la journée (Bloc, 1986). Par ailleurs; au cours de "pipetages" de gouttes de miellat couvrant les feuilles de sapin, nous avons constaté que, avant $10 \mathrm{~h}$, les gouttes de miellat montent facilement par capillarité dans une micropipette; en revanche, lorsque la journée est sèche et ensoleillée, il

* Correspondance et tirés à part 
s'avère impossible de les récolter ainsi, à partir de $11 \mathrm{~h}$ environ.

Nous avons donc étudié :

- l'existence éventuelle d'un rythme circadien d'excrétion du miellat par le puceron, $C$ pectinatae (Nördl) particulièrement exploité par l'abeille domestique dans l'Est de la France;

- l'évolution, au cours de la journée, de la teneur en matière sèche du miellat excrété sur les feuilles de sapin;

- l'existence éventuelle d'une corrélation entre l'hygrométrie et la teneur en matière sèche du miellat;

- l'existence éventuelle d'une corrélation entre le rythme de récolte du miellat par l'abeille et le rythme d'excrétion du miellat par les pucerons et l'évolution de la teneur en eau du miellat au cours de la journée.

\section{MATÉRIEL ET MÉTHODES}

\section{Étude du rythme d'excrétion du miellat}

Le dispositif de récolte du miellat consiste en une plaque ronde de $52 \mathrm{~cm}$ de diamètre mue par un moteur à mouvernent d'horlogerie qui effectue un tour en $24 \mathrm{~h}$. A $5 \mathrm{~cm}$ au-dessus, on dispose une autre plaque ronde, immobile, de $60 \mathrm{~cm}$ de diamètre dans laquelle est découpé un triangle de $10 \mathrm{~cm}$ de base, dont les cótés et le sommet correspondent respectivement au rayon et au centre de la plaque. Ce triangle permet donc un regard sur la plaque toumante dont chaque point est ainsi à découvert pendant $85 \mathrm{~min}$.

Le dispositif est placé de $50 \mathrm{~cm}$ à $1 \mathrm{~m}$ audessous d'une branche de sapin de façon que le regard de la plaque immobile soit a l'aplomb de 1 a 5 pucerons. Vingt-quatre heures plus tard, lors du retrait du dispositif, on contrôle le nombre de pucerons. L'enregistrement n'est pas pris en compte, lorsque des pucerons se sont déplacés.
L'expérience a été aussi réalisée sous des branches d'épicéa, Picea abies L afin de recueillir le miellat excrété par $C$ pilicornis (Hartig), l'un des pucerons qui colonisent l'épicéa.

\section{Étude de la teneur en matière sèche}

Cette mesure est effectuée au moyen de réfractomètres à main qui donnent le pourcentage en matière sèche d'un liquide sucré; ils sont habjtuellement utilisés pour la mesure du pourcentage en eau d'un miel ou d'un jus de fruit. Nous avons utilisé 2 types de réfractomètres : l'un de type Sopelem, permettant des mesures de 50 à $90 \%$ de matière sèche; l'autre de type Atago $\mathrm{N}_{2}$ des mesures de 28 à $62 \%$ de matière sèche.

Deux types de mesures sont effectuées :

1) La mesure du pourcentage en matière sèche du miellat fraîchement excrété. Le miellat étant recueilli en forêt sur des plaques d'aluminium disposées sous un conifère, on pipette une ou plusieurs gouttes, dès qu'elles viennent de tomber, puis on les étale sur le dépoli du réfractomètre. La valeur lue est corrigée en fonction de la température ambiante. Les mesures sont effectuées de 7 à $11 \mathrm{~h}$.

2) La mesure, toutes les heures, de la teneur en matière sèche du miellat couvrant les feuilles de sapin, de 8 à 19 h. A chaque heure, on recueille 4 feuilles de sapin recouvertes de miellat; celuici est ensuite frotté sur le dépoli du réfractomètre, chaque valeur horaire est donc une moyenne de 4 mesures. Le dépoli est nettoyé entre 2 mesures à l'eau distillée, puis à l'alcool à $70^{\circ}$ et enfin, il est séché. Les mesures ont été effectuées au cours de 4 journées du mois d'août 1983 en forêt de Levier (altitude $650 \mathrm{~m}$ ) et 4 journées des mois d'août et de septembre 1984 en forêt de Chaux-Neuve (altitude 1100 m). Il s'agissait de journées sèches et chaudes, à l'exception de 3 journées plus fraîches à passages nuageux, précédées de petits orages nocturnes. Placé en sous-bois, à l'ombre, à $1 \mathrm{~m}$ du sol sous abri, un thermo-hygrographe a permis d'enregistrer en continu la temperature et l'hygrométrie de l'air du 15 août au 15 septembre 1984 (c'est à cette période que les miellées «de sapin » peuvent se produire). 


\section{Mesure de l'activité de récolte du miellat par l'abeille}

Cette étude est faite sur un sapin de 6 à $10 \mathrm{~m}$ de haut, régulièrement visité par des abeilles qui viennent y récolter du miellat. Elle porte précisément sur 4 branches basses qui représentent une aire de 2 a $3 \mathrm{~m}^{2}$.

Toutes les $\mathrm{h}$, nous comptons pendant $10 \mathrm{~min}$ les butineuses qui viennent récolter du miellat sur ces branches. Les comptages sont effectués au cours des journées où il est procédé à l'étude de l'évolution de la teneur en eau du miellat (fig 3). Parallèlement, au cours de 6 journées des mois d'août et de septembre 1981 et 1984 (fig 4), des butineuses sont marquées au moyen de pastilles numérotées au moment de leur première visite sur un jeune sapin de $1,50 \mathrm{~m}$ de haut. Leurs visites sont notées jusqu'à $19 \mathrm{~h}$ : nous avons ainsi cerné le rythme individuel des butineuses quant à la fréquence de leurs récoltes de miellat.

\section{RÉSULTAT}

\section{Le rythme d'excrétion du miellat}

La figure 1 donne le rythme d'excrétion du miellat de $C$ pectinatae et de $C$ pilicornis, à partir de 7 enregistrements de $24 \mathrm{~h}$ réalisés en août et septembre 1984, les mesures étant effectuées de $3 \mathrm{~h}$ en $3 \mathrm{~h}$. On observe ainsi, chez $C$ pectinatae, un maximum d'excrétion entre $20 \mathrm{~h}$ et $24 \mathrm{~h}$ et un minimum entre $12 \mathrm{~h}$ et $15 \mathrm{~h}$ (fig 1A). La comparaison des moyennes d'excrétion grâce à une analyse de variance montre que les différences entre les niveaux d'excrétion dans chaque tranche horaire sont significatives $(F=1,8$ pour 7 et $48 \mathrm{dl})$. Chez $C$ pilicornis, il n'apparaît pas de différences significatives entre les niveaux d'excrétion $(F=14$ pour 7 et $48 \mathrm{dl})$, même si une augmentation apparaît entre 16 et $19 \mathrm{~h}$, suivie d'une diminution jusqu'à $24 \mathrm{~h}$ (fig 1B). II faut observer que, chez cette es- pèce, il existe de grandes variations autour de la moyenne pour chacune des mesures au cours des $24 \mathrm{~h}$.

\section{L'évolution temporelle du pourcentage de matière siche dans le miellat}

\section{Le miellat fraîchement excrété} (10r type de mesures : voir "Matériel et méthodes")

Obtenue à partir de 20 mesures, la proportion de matière sèche dans le miellat oscille entre 52 et $78 \%$. La moyenne étant de $66,5 \%$, le miellat excrété contient donc en moyenne $33,5 \%$ d'eau.
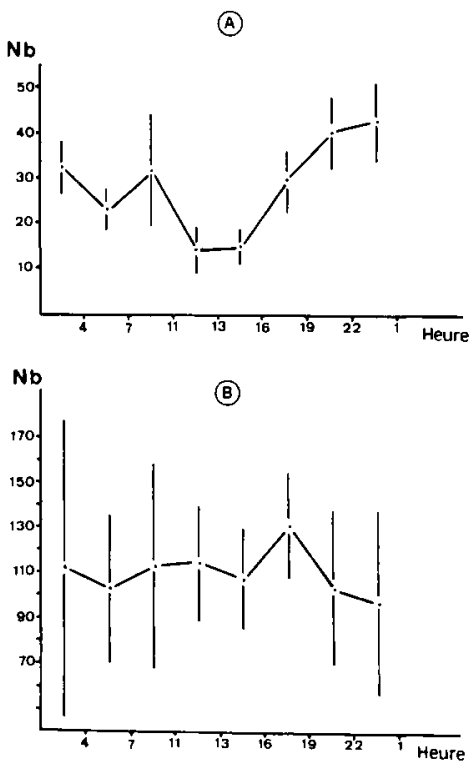

Fig 1. Rythme d'excrétion du miellat (en nombre de gouttes) par Cinara pectinatae (A) (1 a 3 individus) et Cinara pilicomis (B) (30 individus) au cours de 7 enregistrements en continu de $24 \mathrm{~h}$ chacun, effectués à des dates et des lieux différents. Les mesures sont effectuées toutes les $3 \mathrm{~h}$. Chaque moyenne est donnée avec son intervalle de confiance $(P=0,05)$. 


\section{L'influence globale de l'hygrométrie (20 type de mesures : voir «Matériel et méthodes")}

Établie à partir de 51 mesures effectuées en 1984, la courbe de régression de la figure 2 montre qu'il existe une corrélation négative $(r=0,78)$ entre l'hygrométrie relative de l'air et la teneur du miellat en matière sèche. Mais le test de linéarité montre que les écarts des mesures par rapport à la droite de régression linéaire sont trop importants pour considérer la liaison entre l'hygrométrie et la teneur en matière sèche du miellat comme linéaire $(F=$ 6,9 pour 2 et $201 \mathrm{dl}$ ). Les mesures de la teneur en matière sèche du miellat montrent notamment une dispersion importante pour une hygrométrie comprise entre $60 \%$ et $90 \%$. En revanche, pour des valeurs de $10 \%$ à $60 \%$ de l'hygrométrie, les valeurs de la teneur en matière sèche du miellat sont plus groupées.

\section{L'évolution au cours de la journée (2० type de mesures : voir «Matériel et méthodes")}

La figure 3 montre l'évolution journalière de la teneur en matière sèche du miellat (TMS) récolté toutes les heures au cours de 8 journées distinctes $(a, b, c, d, e, f, g$, h). Deux types de courbes se dégagent: le type A (journées a, b, c, d, e et f) et le type $B$ (journées $g$ et $h$ ). Dans le type $A$, on observe une augmentation relativement régulière du pourcentage en matière sèche de $8 \mathrm{~h}$ à $13 \mathrm{~h}$ (c'est à 12-13 h que le pourcentage est le plus élevé : 87 à $90 \%$ ). II apparaît ensuite une phase relativement stable caractérisée par une forte teneur en matière sèche (12 à $16 \mathrm{~h}$ ), suivie d'une baisse jusqu'à $18 \mathrm{~h}$ (le plus souvent aux environs de $80 \%$ ). Ce type de courbes correspond à des journées chaudes et sèches.

Le type de courbes B fait apparaître des fluctuations plus marquées entre les diffé-

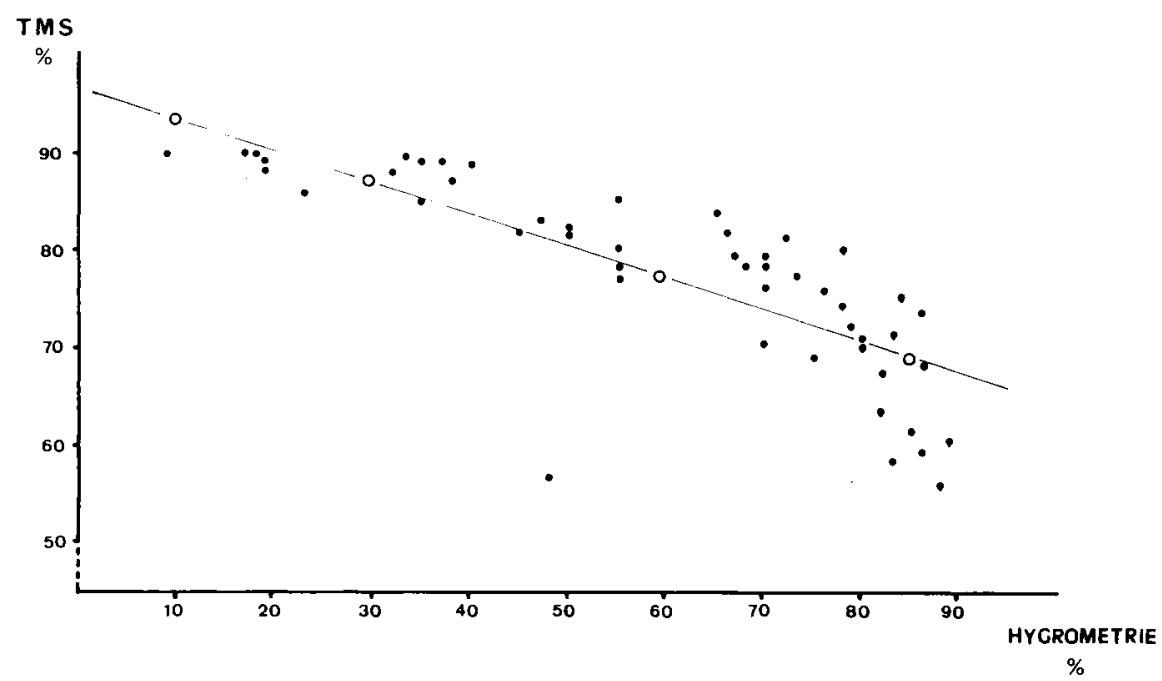

Fig 2. Régression de la teneur en matière sèche du miellat sur l'hygrométrie. La droite de régression linéaire a pour équation $y=97,7-0,34 x$. 
(1)

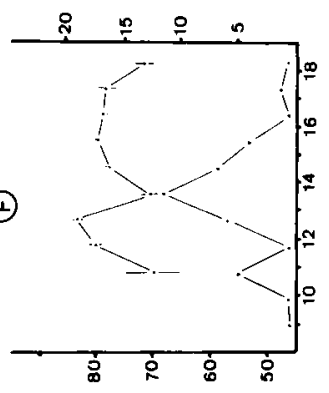

(4)

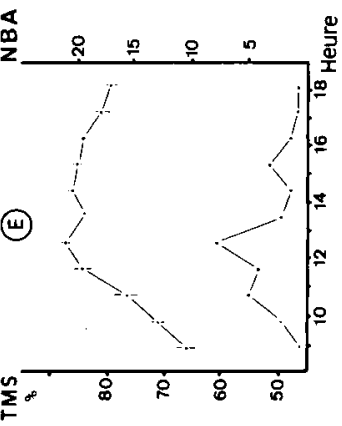

(๑)
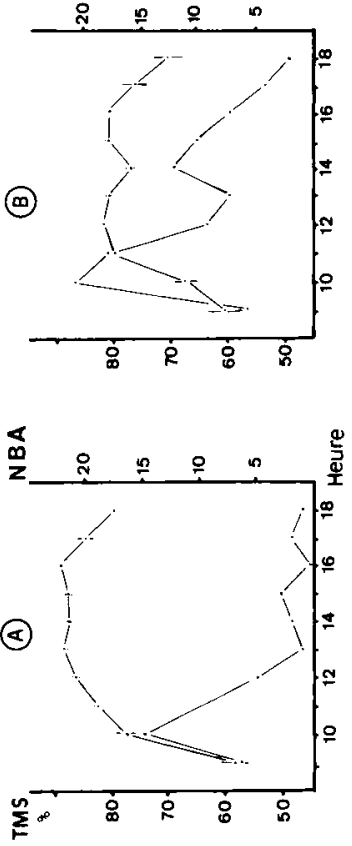

()

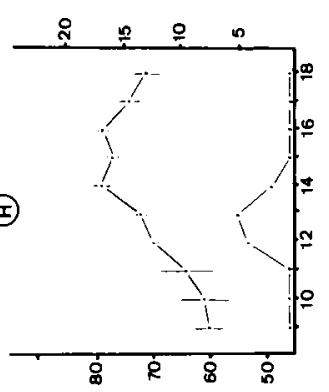

().

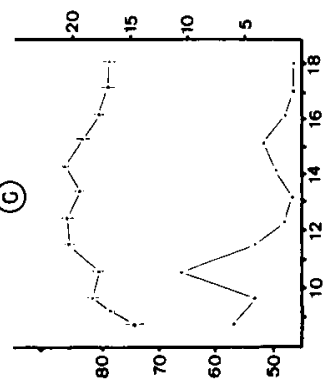

(D)

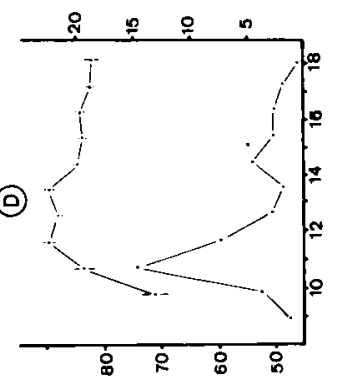

(u)

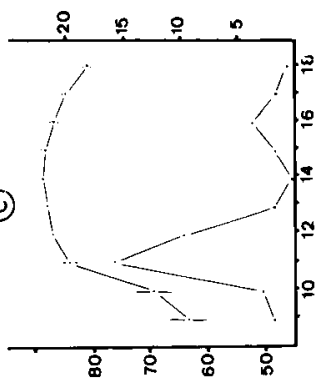

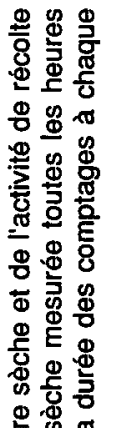

兽

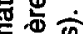

동 ฮ

8

灾

\$ $\$$

온온

도웜

空

ह

궁

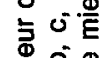

国

ㅇำ के

$\Phi$

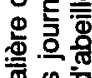

등 웡

흐일

동ㅎㅇ

วิํํำ

ब文

\%

\&

릉음

范。

을

8 严

进完웅

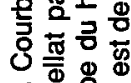

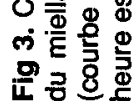


rents moments de la journée (baisse inhabituelle à $14 \mathrm{~h}$ : courbe $\mathrm{g}$; faibles valeurs inhabituelles jusqu'à $13 \mathrm{~h}$ : courbe $\mathrm{h}$ ). Dans ces courbes, les maxima ne dépassent pas 80 ou $85 \%$ de matière sèche. $\mathrm{Ce}$ type de courbes correspond à des journées fraîches et humides, précédées d'un orage nocturne.

Nous pouvons remarquer que, dans les 2 types de courbes, les variations autour de la moyenne sont importantes de 9 à 11 $h$, puis elles sont très faibles ou quasiment nulles de 12 à 16 h; elles augmentent lé- gèrement après $17 \mathrm{~h}$. Les abeilles récolteuses de miellat ont donc, au cours des périodes les plus sèches de la journée, une probabilité de découvrir des miellats ayant le même pourcentage en matière sèche.

\section{Le rythme de récolte du miellat par l'abeille}

Le comptage systématique des abeilles récolteuses de miellat sur le sapin, à raison

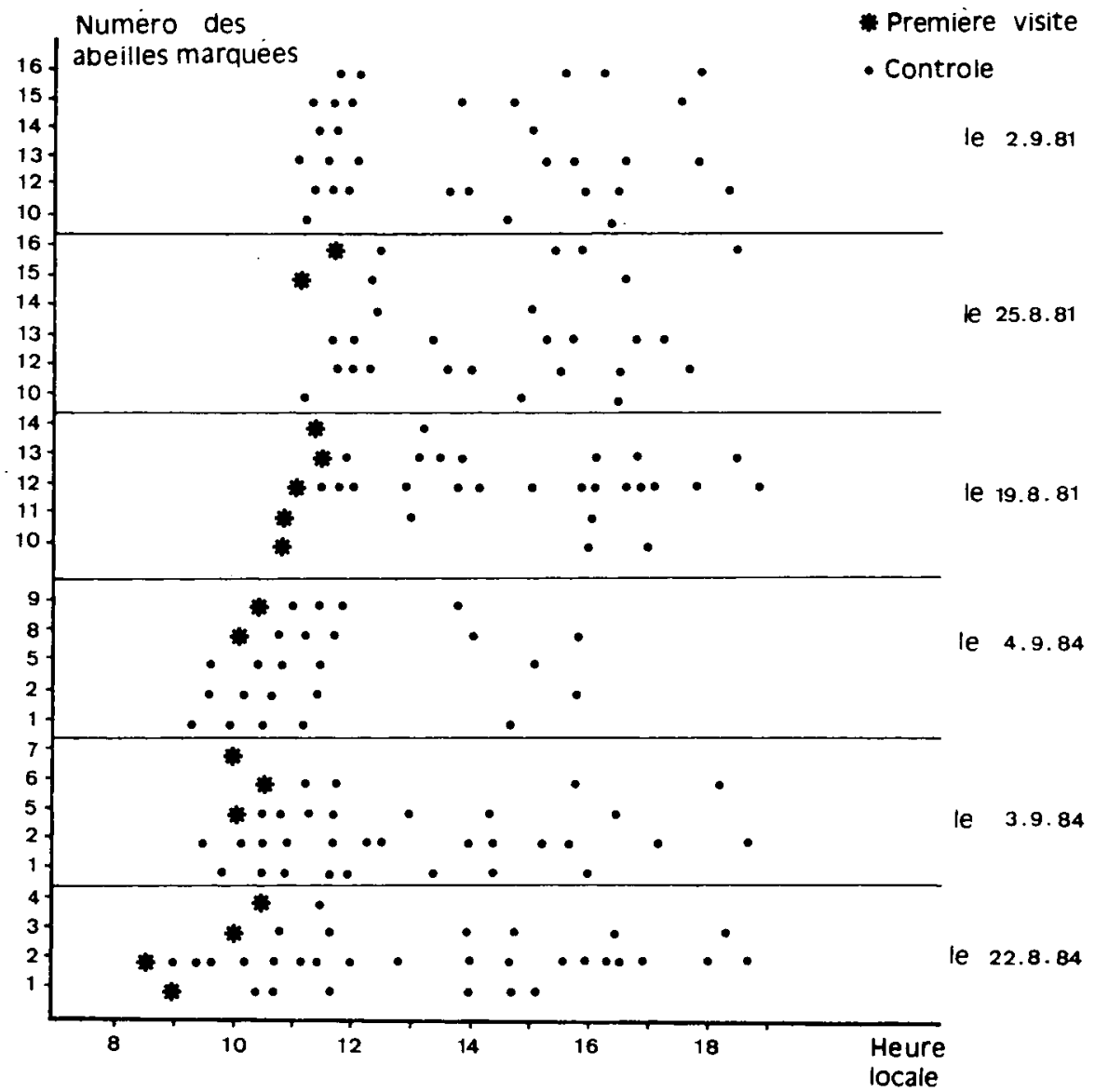

Flg 4. Fréquence des visites individuelles de 16 butineuses marquées sur du miellat de Cinara pectinatae de 8 à 19 h, pendant 3 journées en 1981 (Levier) et 3 journées en 1984 (Chaux-Neuve). 
d'une durée de $10 \mathrm{~min}$ par h pour chaque comptage, révèle un maximum de visites vers $11 \mathrm{~h}$ pour 5 des 6 courbes de la figure 3 ( $a, b, c, d$ et $f)$. On observe ensuite une chute des visites jusqu'à 13 ou $14 \mathrm{~h}$, alors que la teneur du miellat atteint 88 a $90 \%$. Une légère recrudescence des visites apparaît à 15-16 h, mais le nombre de butineuses reste inférieur à 4 par $10 \mathrm{~min}$ d'observation. Dans tous les cas, l'activité de butinage se termine vers $18 \mathrm{~h}$ ou un peu plus tard.

En revanche, les courbes e, $g$ et $h$ présentent un pic d'activité déplacé vers 13 ou $14 \mathrm{~h}$. Or, les courbes $\mathrm{g}$ et $\mathrm{h}$ correspondent à des journées caractérisées par des conditions météorologiques particulières : orages nocturnes au cours de la nuit précédente (courbe g), faibles températures et ciel couvert tout au long de la journée (courbe h).

La figure 4 montre que le nombre maximal de visites observées à un emplacement donné, de 8 à 19 h, par l'abeille $n^{\circ} 2$ est de 19 alors que le nombre moyen de visites pour les abeilles observées est de 6. L'examen de cette figure permet aussi de déduire que certaines abeilles exploitent régulièrement la même source de nourriture pendant une longue période (butineuses habituelles). Ainsi sur 29 abeilles marquées en 1981 et 34 en 1984, 6 (numéros $10,12,13,14,15$ et 16) et 3 (numéros 1,2 et 5) sont venues récolter du miellat au même emplacement pendant respectivement 14 et $13 \mathrm{j}$. En revanche, d'autres ne le font qu'occasionnellement, à des intervalles de temps irréguliers (butineuses occasionnelles).

Construite à partir de la figure 4 , la figure 5 montre comment évolue le rythme de récolte. Elle confirme l'existence d'un maximum de visites vers $11-12 h$, puis d'un minimum de visites entre 12 et $14 \mathrm{~h}$. Une nouvelle augmentation apparaît entre 14 et $17 \mathrm{~h}$.

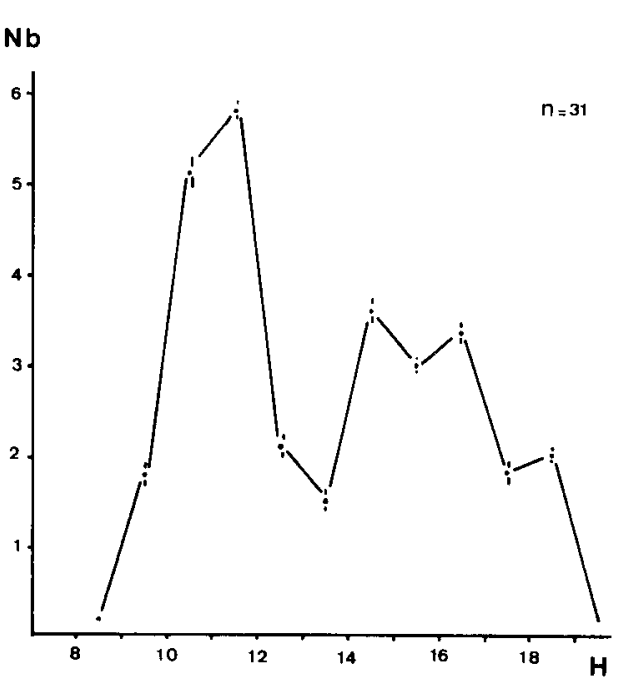

Fig 5. Rythme moyen de récolte du miellat de Cinara pectinatae par l'abeille $(N$ de visiteuses $=$ 31) au cours de la journée, de 8 à $19 \mathrm{~h}$.

\section{DISCUSSION}

Nos résultats montrent qu'il existe un rythme d'excrétion du miellat par $C$ pectinatae avec un maximum nocturne de 20 à $24 \mathrm{~h}$ et un pic matinal de 7 à $10 \mathrm{~h}$; le minimum de l'excrétion apparaît de 12 à $15 \mathrm{~h}$. Ces résultats sont en accord avec ceux de Rihar (1963) et de Maquelin (1974). Le miellat fraîchement excrété a un pourcentage moyen de $66 \%$ de matière sèche, ce qui est conforme aux données de Liebig (1977). Cependant, la teneur en matière sèche varie beaucoup au cours de la journée : le miellat perd de l'eau et devient très concentré à partir de 11 ou $12 \mathrm{~h}$, en relation avec la baisse de l'hygrométrie de l'air (la teneur en matière sèche devient alors supérieure à $85 \%$ ).

Étudié au moyen de 2 méthodes différentes, le rythme de récolte du miellat par l'abeille montre que les visites atteignent la fréquence la plus élevée de 10 à 12 h et la 
plus faible de 13 à $14 \mathrm{~h}$. La récolte reprend ensuite au cours de l'après-midi, mais avec une fréquence beaucoup plus faible que le matin. Le rythme de récolte du miellat par l'abeille apparaît donc en relation avec le pourcentage en matière sèche du miellat, la relation étant plus marquée au cours des journées sèches et chaudes. Nos données confirment les observations de Rihar (1977) et de Demianowicz (1978) qui, mesurant le poids et le pourcentage en matière sèche du miellat contenu dans le jabot des butineuses revenant à la ruche, observe une corrélation négative entre ces 2 paramètres. Demianowicz (1978) montre notamment que, entre 11 et $13 \mathrm{~h}$, le pourcentage en matière sèche du miellat récolté est élevé, tandis que le poids du miellat est faible. Or, c'est à ce moment que les butineuses récoltent beaucoup d'eau. On peut donc logiquement faire l'hypothèse que la baisse du poids du miellat entre 11 et $13 \mathrm{~h}$ est due à une déshydratation du miellat et que l'augmentation de la récolte d'eau par les butineuses vise, au moins partiellement, à la réhydratation du miellat absorbé.

Par conséquent, de même qu'il y a une relation entre la concentration du nectar dans les plantes à fleurs et l'activité de récolte de l'abeille (Butler, 1944; Shuel, $1955,1964)$, le rythme de récolte du miellat de $C$ pectinatae par l'abeille apparaît corrélé avec l'évolution de la teneur en eau du miellat. Par comparaison, au cours des fortes miellées, la récolte du nectar ne présente pas de fluctuations significatives au cours de la journée, au moins lorsqu'aucune perturbation climatique n'intervient (Lundie, 1925), sauf en milieu de la journée où elle diminue (Mesquida et Renard, 1982). Certains biologistes expliquent cette diminution de la mi-journée par une baisse de sécrétion nectarifère, alors que, pour d'autres, elle serait due à la diffi- culté des abeilles à danser lorsque le soleil est très proche du zénith.

Cependant, on n'observe pas de relation causale simple entre le rythme d'excrétion du miellat par le puceron $C$ pectinatae et le rythme de récolte de ce miellat par l'abeille. En effet, c'est au moment où le miellat s'assèche progressivement entre 10 et $12 h$, après une excrétion maximale au cours de la nuit, que l'activité de récolte du miellat par l'abeille est maximale. On peut donc penser que les conditions d'hygrométrie et de température de la nuit et de la matinée permettent au miellat de conserver des caractéristiques telles qu'il peut être récolté sans difficulté par les abeilles, dès que les activités journalières de butinage commencent. En revanche, entre 12 et $14 \mathrm{~h}$, les abeilles interrompent leur récolte, alors que la teneur du miellat en matière sèche atteint un maximum.

Summary - The relation between excretory rhythm and water content of honeydew from Cinara pectinatae Nördl and Cinara pilicornis (Hartig) and the honeydew harvesting activity of the honeybee, Apis mellifica L. Preliminary observations of Cinara pectinatae honeydew harvesting by honey bees show that honeydew harvesting activity varies throughout the day. The honeydew excretion activity of Cinara pectinatae and Cinara pilicornis was studied using a revolving plate that allowed honeydew droplets excreted in $24 \mathrm{~h}$ to be counted. Maximum excretion occurs between 2000 and $2400 \mathrm{~h}$ and a minimum excretion between 1200 and 1500 in $C$ pectinatae (fig 1A). In $C$ pilicornis there does not appear to be a circadian rhythm of honeydew excretion (fig 1B). Refractometer measurements of the proportion of honeydew dry matter show that the dry matter content of freshly excreted honeydew varies between $52 \%$ and 
$78 \%$. We also found a negative correlation between the relative hygrometry and the honeydew dry matter content (fig 2). It was noted that dry matter content increases during the morning until $1200 \mathrm{~h}$, is stable during the early afternoon at a high value and decreases after $1600 \mathrm{~h}$ (fig 3). The study of honeydew harvesting activity shows that honey bees collect honeydew at a high level from $1000-1100 \mathrm{~h}$ during warm and dry weather (fig $3 a, b, c, d, f$ ) and from $1200-1400 \mathrm{~h}$ during raw weather (fig $3 \mathrm{~g}, \mathrm{~h}$ ). Thus, the drying of the honeydew during the day and the honeydew excretion rhythm are 2 factors which apparently limit the harvesting of honeydew by the honey bee.

Cinara / aphid / honeydew / excretory rhythm / harvesting rhythm / Apis mellifica

\section{Zusammenfassung - Die Beziehungen} zwischen Exkretionsrhythmus und Wassergehalt des Honigtaues der Aphiden Cinara pectinatae und $C$ pilicornis und dem Sammelrhythmus der Honigbiene Apis mellifera L. Vorläufige Beobachtungen des Sammelns der Honigbienen am Honigtau von Cinara pectinatae zeigte über den ganzen Tag beträchtliche Schwankungen der Sammelaktivität. Die Studien ergaben folgende Beziehungen zwischen dem Sammelrhythmus der Bienen, der Abscheidung der Aphiden und dem Gehalt des Honigtaus an Trockensubstanz:

Mittels einer sich langsam drehenden Platte wurden die Exkret-Tropfen über $24 \mathrm{~h}$ einzeln aufgefangen. Bei Cinara pectinatae ergab sich ein ExkretionsMaximum zwischen 20.00-24.00 h und ein Minimum zwischen 12.00-15.00 h (Abb 1A). Bei Cinara pilicornis scheint kein derartiger circadianer Rhythmus der
Honigtau-Exkretion zu bestehen (Abb 1B).

Die Bestimmung der Trockensubstanz des Honigtaus mittels eines Refraktometers zeigte:

- der Anteil der Trockensubstanz frisch abgesonderten Honigtaues schwankte zwischen $52 \%-78 \%$;

- es besteht eine negative Korrelation zwischen der relativen Luftfeuchtigkeit und dem Gehalt an Trockensubstanz (Abb 2);

- der Gehalt an Trockensubstanz steigt während der Morgenstunden bis $12.00 \mathrm{~h}$, bleibt während des Nachmittags auf einem hohen Niveau stabil und sinkt nach $16.00 \mathrm{~h}$ ab (Abb 3).

Die Untersuchung des Sammelrhythmus der Honigbienen durch Zählen der an Fichtenzweigen sammelnden Bienen, entweder $10 \mathrm{~min}$ lang in jeder Stunde (Abb 3) oder durch Zählen einzelner Besuche 16 markierter Bienen zwischen $8.00 \mathrm{~h}$ und $19.00 \mathrm{~h}$ (Abb 4) zeigte, daß die Honigbienen mit großer Intensität bei warmem und trockenem Wetter zwischen $10.00 \mathrm{~h}-11.00$ sammeln (Abb 3 a, b, c, d, f), bei rauhem Wetter aber zwischen $12.00 \mathrm{~h}$ und $14.00 \mathrm{~h}$ (Abb 3 $g, h)$.

Es hat demnach den Anschein, daß das Eintrocknen des Honigtaues während des Tages und der Rhythmus der Honigtauexkretion die beiden Faktoren sind, welche das Einsammeln des Honigtaus durch die Bienen begrenzen.

\section{Cinara / Aphiden / Honigtau / Exkretionsrythmus / Sammelrhythmus / Apis mellifera}

\section{RÉFÉRENCES}

Bloc A (1986) Étude éco-éthologique de l'explojtation des miellats du Puceron Cinara pectinatae (Nördl) (Homoptera-Lachnidae) par 
l'abeille domestique (Apis mellifica $\mathrm{L}$ ). Thèse, université de Franche-Comté, $171 \mathrm{p}$

Butter CG (1944) The influence of various physical and biological factors of the environment of the honeybee activity. An examination of the relationship between activity and nectar concentration and abundance. J Exp Biol 5, 5-12

Demianowicz Z (1978) Inhalt der Honigblase der Arbeitsbienen von Apis mellifica $L$ während der Tannenhonigtautracht. Apidologie 9, 117-122

Kleber E (1935) Hat das Zeitgedächtnis der Bienen biologische Bedeutung ? Z Vgl Physiol 22, 211-262

Körner I (1939) Zeitgedächtnis und Alarmierung bei den Bienen. $Z$ Vgl Physiol 27, 445-459

Liebig G (1979) Hinweise für die Beobachtung der Tannentracht. Allg Dsch Imkerzeitung IV, 204-206

Lundie AE (1925) The flight activities of the honeybee. US Dep Agric Agric Inform Bull 1-37
Maquelin C (1974) Observations sur la biologie et l'écologie d'un puceron utile à l'apiculture, Buchneria pectinatae. Thèse, Zurich

Mesquida J, Renard M (1982) Remarques sur l'activité de butinage des abeilles. Bull Tech Apic 9, 9-20

Ribbands CR (1953) The behaviour and social life of honeybees. Daver, New York, $352 p$

Rihar J (1963) Einfluss der aüßeren Faktoren auf die Konzentration des Tannenhonigtaues. $X I X$ Apimondia Kongress Prag, 648-651

Rihar J (1977) Reaktion von Honigbienen auf die Honigtauabgabe der grünen Tannenhoniglaus, Buchneria pectinatae (Nördl). Apidologie 8, 413-418

Suhuel RW (1955) Nectar secretions in relation to nitrogen supply, nutritional status, and growth of the plant. Can J Agric Sci 35, 124138

Suhuel RW (1964) L'influence des facteurs externes sur la production du nectar. Ann Abeille (Paris) 7, 5-12 